\title{
Effect of rotator cuff dysfunction on the initial mechanical stability of cementless glenoid components
}

\author{
Daniel R. Suárez • Edward R. Valstar · \\ Jacqueline C. van der Linden · Fred van Keulen • \\ Piet M. Rozing
}

Received: 5 July 2008/ Accepted: 3 March 2009/Published online: 21 March 2009

(C) The Author(s) 2009. This article is published with open access at Springerlink.com

\begin{abstract}
The functional outcome of shoulder replacement is related to the condition of the rotator cuff. Rotator cuff disease is a common problem in candidates for total shoulder arthroplasty; this study relates the functional status of the rotator cuff to the initial stability of a cementless glenoid implant. A 3D finite element model of a complete scapula was used to quantify the effect of a dysfunctional rotator cuff in terms of bone-implant interface micromotions when the implant is physiologically loaded shortly after surgery. Four rotator cuff conditions (from fully intact to progressively ruptured rotator cuff tendons) as well as two bone qualities were simulated in a model. Micromotions were significantly larger in the worst modeled cuff dysfunction (i.e. the supraspinatus and infraspinatus tendons were fully dysfunctional). Micromotions were also
\end{abstract}

D. R. Suárez $(\bowtie) \cdot$ E. R. Valstar

Biomechanics and Imaging Group, Department of Orthopaedics,

Leiden University MC, Leiden, The Netherlands

e-mail: d.r.suarez_venegas@lumc.nl

D. R. Suárez · J. C. van der Linden · F. van Keulen

Department of Precision and Microsystems Engineering, Faculty of Mechanical, Maritime, and Material Engineering, Delft University of Technology, Delft, The Netherlands

E. R. Valstar

Department of Biomechanical Engineering,

Faculty of Mechanical, Maritime, and Material Engineering,

Delft University of Technology, Delft, The Netherlands

J. C. van der Linden

Orthopaedic Research Laboratory, Erasmus MC,

Rotterdam, The Netherlands

P. M. Rozing

Department of Orthopaedics, Leiden University MC,

Leiden, The Netherlands significantly different between conditions with healthy and poor bone quality. The implant's initial stability was hardly influenced by a dysfunctional supraspinatus alone. However, when the infraspinatus was also affected, the glenohumeral joint force was displaced to the component's rim resulting in larger micromotions and instability of the implant.

Keywords Rotator cuff - Cementless - Modeling · Micromotions - Total shoulder arthroplasty (TSA) . Glenoid

$\begin{array}{ll}\text { Abbreviations } & \\ \text { HB } & \begin{array}{l}\text { Bone quality in healthy bone } \\ \text { Bone quality in RA bone } \\ \text { Delft Shoulder and Elbow } \\ \text { Model } \\ \text { YSEM }\end{array} \\ E & \begin{array}{l}\text { Foung's Modulus } \\ \text { Finite Element }\end{array} \\ \text { FE } & \text { Glenohumeral Joint Reaction } \\ \text { JRF } & \text { Force } \\ \text { Inter-Quartile Range } \\ \text { RA } & \begin{array}{l}\text { Rheumatoid Arthritis } \\ \text { Specific rotator cuff clinical } \\ \text { S100, S50, S00, SINF00 } \\ \text { conditions in this study } \\ \text { TSA }\end{array} \\ v & \begin{array}{l}\text { Total Shoulder Arthroplasty } \\ \text { Poisson's ratio }\end{array}\end{array}$

\section{Introduction}

Total shoulder arthroplasty (TSA) have been good in terms of pain relief and improvement in range of motion and function. However, the prognostic implications of rotator cuff disease and bone stock on the clinical outcome of TSA 
(i.e. glenoid component loosening) are still a matter of concern.

A diseased rotator cuff is a common problem in patients qualifying for a TSA; a diseased cuff may present tears and/or fatty degeneration in one or more of its tendons. If tears are found during surgery, an attempt will be made to repair the tendons. If repair is not possible a glenoid component should not be implanted [9]. A degenerated rotator cuff has a negative effect on the clinical outcome of shoulder replacement, i.e. reduced Constant score, range of motion, and a lower subjective satisfaction score [7, 11, 21].

Poor bone stock and poor bone quality like in rheumatoid arthritis (RA) patients are also considered as risk factors for the use of a glenoid component. The question is: can a dysfunctional rotator cuff and diseased bone still support a glenoid component in TSA without the risk for increased micromotions with subsequent early loosening. The latter is of importance since TSA is sometimes the only alterative when a deficient glenoid is present, or because the surgeon expects superior clinical results (pain as well as range of motion) as compared with a hemiarthroplasty.

For cementless glenoid components, initial mechanical stability is highly relevant for their mid- and long-term fixation via bone ingrowth. Excess of relative motion between the implant and the bone will inhibit bone ingrowth completely $[17,18]$. The aim of this study is to quantify the initial mechanical stability of a cementless glenoid implant in presence of a progressive dysfunction of the rotator cuff, firstly in a scapula with healthy bone quality, and secondly in a scapula with poor bone quality. Dysfunction of the rotator cuff is represented in this study by a reduction of supraspinatus and infraspinatus muscle forces separately and in combination, and poor bone quality is modeled by means of a reduction of the bone stiffness in the scapula.

\section{Methods}

The initial mechanical stability of a cementless glenoid implant was studied by calculating the micromotions at the bone-implant interface resulting from physiological loads. A three-dimensional finite element (FE) model of a complete scapula with an implanted cementless glenoid component was used to determine the interface micromotions shortly after surgery.

\subsection{Modeling of the rotator cuff dysfunction}

Deficiency of the rotator cuff was simulated by reduction of the muscle forces of the supraspinatus and infraspinatus, from $100 \%$ (healthy) to $0 \%$ (fully dysfunctional). Four different rotator cuff conditions were considered: with healthy rotator cuff tendons which are able to exert a maximum of $100 \%$ of the capable force (S100); with supraspinatus exerting a maximum of $50 \%$ of its capable force (S50); with supraspinatus exerting $0 \%$ of its capable force (SO0); and with supraspinatus and infraspinatus exerting both $0 \%$ of their capable force (SINF00). Larger rotator cuff dysfunctions were not considered. Here, capable force refers to the maximum force that a healthy muscle is able to produce and which depends on the muscle cross-sectional area and its current length (which depends on the arm position).

To postoperatively represent upper arm motions, four static positions at $0^{\circ}, 30^{\circ}, 60^{\circ}$ and $90^{\circ}$ relative to the trunk in either abduction (on the coronal plane) or forward flexion were modeled. The Delft Shoulder and Elbow Model (DSEM) was used to determine all the muscle and joint forces (magnitude, orientation, and application point(s)/areas) on the scapula for each one of studied arm positions. This model uses pre-registered motions of the bones and external loading as input, and calculates muscle and joint reaction forces and moments using an optimization method (i.e. minimization of the sum of squared muscle stresses) [22]. In case a glenohumeral joint reaction force (JRF) was predicted out of the glenoid, as it may happen with a large dysfunctional rotator cuff, the application point of this force was translated to the closest point on the glenoid rim. No subluxation was simulated. Finally, when the insertion points or areas defined in the DSEM did not correspond with the nodes in the FE model, the muscle forces were shifted to the nearest nodes and slightly altered according to a minimization formulation in order to retain equilibrium.

In the DSEM the force exerted by each muscle can be limited; this allowed us to calculate the loading conditions for the different rotator cuff conditions in all the studied arm positions; similar procedure was used by Magermans et al. [13]. The JRF was one of the forces predicted by the DSEM and it is used as an input to the FE model, however, here it is also discussed how its change could influence the interface micromotions of the different studied cases. The FE model of the present study and the DSEM used the same cadaveric scapula; this ensured that the joint reaction and muscle forces were closer to the real physiological forces than with the ones given by theoretical force profiles.

2.2 Characteristics of the model and definition of micromotions

The scapula and implant were both modeled as linear elastic materials. The FE models consisted of approximately 
310.000 linear tetrahedra with an approximate edge length of $1 \mathrm{~mm}$ for elements in the glenoid and prosthesis and $2 \mathrm{~mm}$ for other regions. The coefficient of friction at the bone-implant interface was set conservatively to 0.5 .

Micromotions were defined as the change in distance between the nodes at the glenoid side and the implant side of the interface when the implant and the scapula were loaded with the glenohumeral joint reaction and muscle forces. Normal and tangent components of the micromotions with respect to the interface were both taken into account. Micromotions were calculated at each node at the interface for each arm position. Mechanical analysis was done using Full Newton-Raphson and a Coulomb friction model in MSC.marc (version 2005r2, Palo Alto, USA).

\subsection{Geometry and material properties: scapula}

The geometry of a cadaveric scapula was based on a CTdata set with a resolution of $0.33 \times 0.33 \times 0.5 \mathrm{~mm}$, which was first reconstructed by Kaptein and van der Helm [12]. The Young's modulus of the scapula was obtained through its relationship with the bone density and the image's Hounsfield Units. The relations between the Young's modulus and the apparent density for trabecular and cortical bone were taken from the works done by Rice et al. [19] and by Schaffler and Burr [20], respectively.

The resultant Young's moduli ranged from 70 to $2200 \mathrm{MPa}$ for trabecular bone and from 2200 to $15200 \mathrm{MPa}$ for cortical bone. Furthermore, the elements along the surface of the scapula were considered to be cortical bone and their Young's modulus was set to the maximal value, $15200 \mathrm{MPa}$. The Poisson's ratio was set to 0.3 for the entire scapula.

To determine the effect of inferior bone quality on the studied variables, Young's moduli were reduced to $50 \%$ and $10 \%$ of their original value for cortical and trabecular bone, respectively. This reduction can be associated with rheumatoid arthritis (RA) where the bone has a considerably inferior bone quality. This was done following the work done by Dalstra et al. [5] and Frich [10]. HB will be used for healthy bone and RAB will refer to RA bone. Table 1 shows the eight studied cases.

\subsection{Geometry and material properties: implant}

The cementless glenoid implant used in this study was a round-shaped and fully conforming component. The glenoid component had a metal back with a porous coating made of a CoCrMo alloy, a polyethylene inlay and a single central screw (ESKA Implants, Lübeck, Germany). This implant has been used clinically in our institution. The porous coating was simulated as a continuous layer of material with apparent material properties equivalent to the real properties of the coating. A perfect fit between the implant's metal backing and the bone was assumed. The effect of the screw was simulated by tying the interface nodes where the screw is located at the component's metal back. The Young's moduli (E) for the solid back and porous coating were $225 \mathrm{GPa}$ and $3.5 \mathrm{GPa}$, respectively. The Poisson's ratio $(v)$ was 0.3 in both cases. The material properties for the polyethylene cup were: $\mathrm{E}=1.174 \mathrm{GPa}$ and $v=0: 46$.

Virtual implantation of the cementless glenoid component was done with an imaging processing/visualization platform, DeVIDE [3]. The implant was placed in a neutral alignment with respect to the original glenoid, so surfaces of the glenoid and component coincided. Geometric subtraction of the prosthesis mesh from the scapula mesh was done with the GTS package (http://gts. sourceforge.net/). The final configuration was inspected and approved by an expert surgeon (PMR). After subtraction, surface re-meshing and solid meshing of the scapula surface were done with MSC.Patran (version 2005, Palo Alto, USA).

Table 1 Rotator cuff conditions and bone qualities in this study

\begin{tabular}{|c|c|c|c|c|}
\hline Case & Bone quality & Rotator cuff condition & $\begin{array}{l}\text { Clinical description of the rotator } \\
\text { cuff state }\end{array}$ & How it is modeled in the present study \\
\hline 1 & HB & \multirow[t]{2}{*}{ S100 } & \multirow[t]{2}{*}{ Healthy tendons } & \multirow{2}{*}{$\begin{array}{l}\text { All the rotator cuff muscles exerting } \\
100 \% \text { of their capable force }\end{array}$} \\
\hline 2 & RAB & & & \\
\hline 3 & HB & \multirow[t]{2}{*}{ S50 } & \multirow[t]{2}{*}{ Partial failure of the supraspinatus } & \multirow{2}{*}{$\begin{array}{l}\text { Reduction of the capable force of the } \\
\text { supraspinatus to } 50 \%\end{array}$} \\
\hline 4 & RAB & & & \\
\hline 5 & HB & \multirow[t]{2}{*}{ S00 } & \multirow[t]{2}{*}{ Total failure of the supraspinatus } & \multirow{2}{*}{$\begin{array}{l}\text { Supraspinatus does not exerts force on the } \\
\text { scapula, i.e. reduction to } 0 \%\end{array}$} \\
\hline 6 & RAB & & & \\
\hline 7 & HB & \multirow[t]{2}{*}{ SINF00 } & \multirow{2}{*}{$\begin{array}{l}\text { Total failure of supraspinatus and } \\
\text { infraspinatus }\end{array}$} & \multirow{2}{*}{$\begin{array}{l}\text { Supraspinatus and infraspinatus do not exer } \\
\text { forces on the scapula }\end{array}$} \\
\hline 8 & RAB & & & \\
\hline
\end{tabular}

Except for the supraspinatus and infraspinatus, all the other muscles that act on the scapula were able to exert their maximum capable force if that was necessary. HB and RAB refer to the two bone qualities simulated in this study: healthy and rheumatoid arthritic (RA) bone, respectively 


\subsection{Presentation of the results and statistics}

The calculated interface micromotions are presented using box plots. A box plot contains information about the median value (middle line), interquartile range IQR (upper and lower limits of the box, i.e. the 25 th and the 75 th percentiles), and the maximum and minimum values, excluding the outliers (whisker). The median and IQR give information about the location and the dispersion of the micromotions at the bone-implant interface. Notches display the variability of the median between the samples. The width of a notch is computed so that box plots whose notches do not overlap have different medians at the $5 \%$ significance level.

Additionally, a nonparametric one-way analysis of variance (Kruskal-Wallis test $\mathrm{p}<0.01$ ) and a multiple comparison procedure (Tukey-Kamer method, $\alpha=0.05$ ) were performed to study the differences between the studied cases.

\section{Results}

Estimation of the stability of the implant was done in terms of the interface micromotions. These were calculated for each upper arm position (i.e. abduction and forward flexion at $0^{\circ}, 30^{\circ}, 60^{\circ}$ and $90^{\circ}$ ), rotator cuff condition (S100, S50, S00, and SINF00), and bone quality (HB and RAB).

\subsection{The glenohumeral joint reaction force, magnitude and application points}

The glenohumeral joint reaction force (JRF) as calculated with the DESM changed due to the changes in the rotator cuff condition (i.e. changes in the muscle forces). Table 2 and Fig. 1 show magnitudes and application points of the JRF for all the models. FE models with RA bone (RAB) had the same loading conditions as the models with healthy bone (HB). The JRF increased with rising of the arm with a maximum at $90^{\circ}$ in either arm abduction or forward flexion. The JRF magnitude slightly changed for the different rotator cuff conditions but its application point on the glenoid did change. A displacement of the application point to the component's rim when supraspinatus and infraspinatus muscles were fully dysfunctional (SINF00) was evident during forward flexion.

\subsection{Interface micromotions in different arm positions}

Different arm positions resulted in different levels of interface micromotions. Arm forward flexion always caused larger interface micromotions than arm abduction, and the largest micromotions were found when the arm was
Table 2 Glenohumeral joint reaction force (JRF) for each rotator cuff condition and different arm positions

\begin{tabular}{lllll}
\hline \multirow{5}{*}{} & \multicolumn{3}{l}{ Glenohumeral Joint Reaction Force $[\mathrm{N}]$} \\
\cline { 2 - 5 } & \multicolumn{2}{l}{ Abduction } & & \\
\cline { 2 - 4 } & $0^{\circ}$ & $30^{\circ}$ & $60^{\circ}$ & $90^{\circ}$ \\
\hline Rotator cuff condition & & & \\
S100 & 93 & 233 & 359 & 427 \\
S50 & 92 & 234 & 364 & 441 \\
S00 & 92 & 234 & 363 & 441 \\
SINF00 & 114 & 249 & 344 & 427 \\
Forward flexion & & & & \\
S100 & 76 & 240 & 353 & 421 \\
S50 & 79 & 239 & 359 & 421 \\
S00 & 79 & 239 & 350 & 421 \\
SINF00 & 93 & 250 & 364 & 418 \\
\hline
\end{tabular}

Rotator cuff state S100: healthy tendons and, subsequently, exerting a maximum $100 \%$ of the capable force; S50, supraspinatus exerting a maximum $50 \%$ of its capable force; S00, supraspinatus exerting $0 \%$ of its capable force; and SINF00, supraspinatus and infraspinatus exerting $0 \%$ of their capable force

positioned at $60^{\circ}$ in forward flexion. In general, the variation between rotator cuff conditions was smaller than the variation between the different arm positions for the same cuff conditions; see Fig. 2.

\subsection{General comparison between the studied cases}

We made sets of the results for every studied case, one case being defined as the combination of one rotator cuff condition and one bone quality. These sets consisted of the largest micromotions during all the considered arm positions at each node of the bone-implant interface for every studied case. Because these samples were not normally distributed we used the median and the IQR values to compare the interface micromotions between the different studied cases. Histograms for two studied cases are plotted in Fig. 3. Medians and IQR values for the studied cases are graphically displayed in Fig. 4.

The results showed a significant difference in the interface micromotions between the models with healthy (HB) and with RA bone (RAB). Medians of the interface micromotions when RA bone was simulated were always the largest; see Fig. 4. Furthermore, the median slightly increased when there was dysfunction of the rotator cuff tendons in both healthy and RA bone. A Kruskal-Wallis test ( $\mathrm{p}<0.01$ ) followed by a multicomparison test (TukeyKamer method, $\alpha=0.05$ ) revealed a significant difference between micromotions of the cases that have a complete failure of supraspinatus and infraspinatus (SINF00) and all the other studied cases, with exception of the case HB-S00 that is not significantly different from the case HB-SINF00. 
Fig. 1 Application points of the glenohumeral joint reaction force (JRF) on the (prosthetic) articular surface for the four different rotator cuff states (S100, S50, S00, and SINF00) in arm abduction (a) and forward flexion (b). Filled markers represent the application point when the arm is at $0^{\circ}$. The other points, united by lines, are the following arm positions $\left(30^{\circ}, 60^{\circ}\right.$ and $\left.90^{\circ}\right)$. The left side of the circles is the posterior side of the glenoid

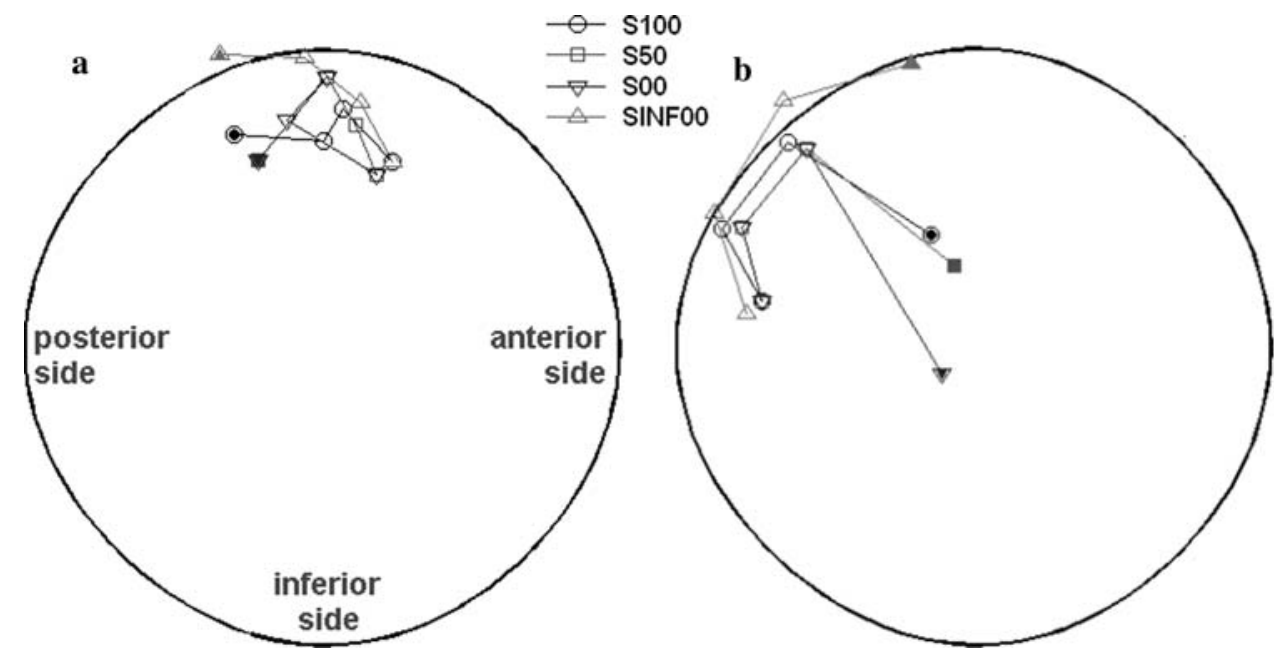

Fig. 2 Box plot of the interface micromotions in each arm position for different rotator cuff conditions with healthy bone (HB). The number after the dot in the rotator cuff state refers to the arm position in degrees. For brevity, S50, which had very similar results to $\mathrm{S} 100$, was not included

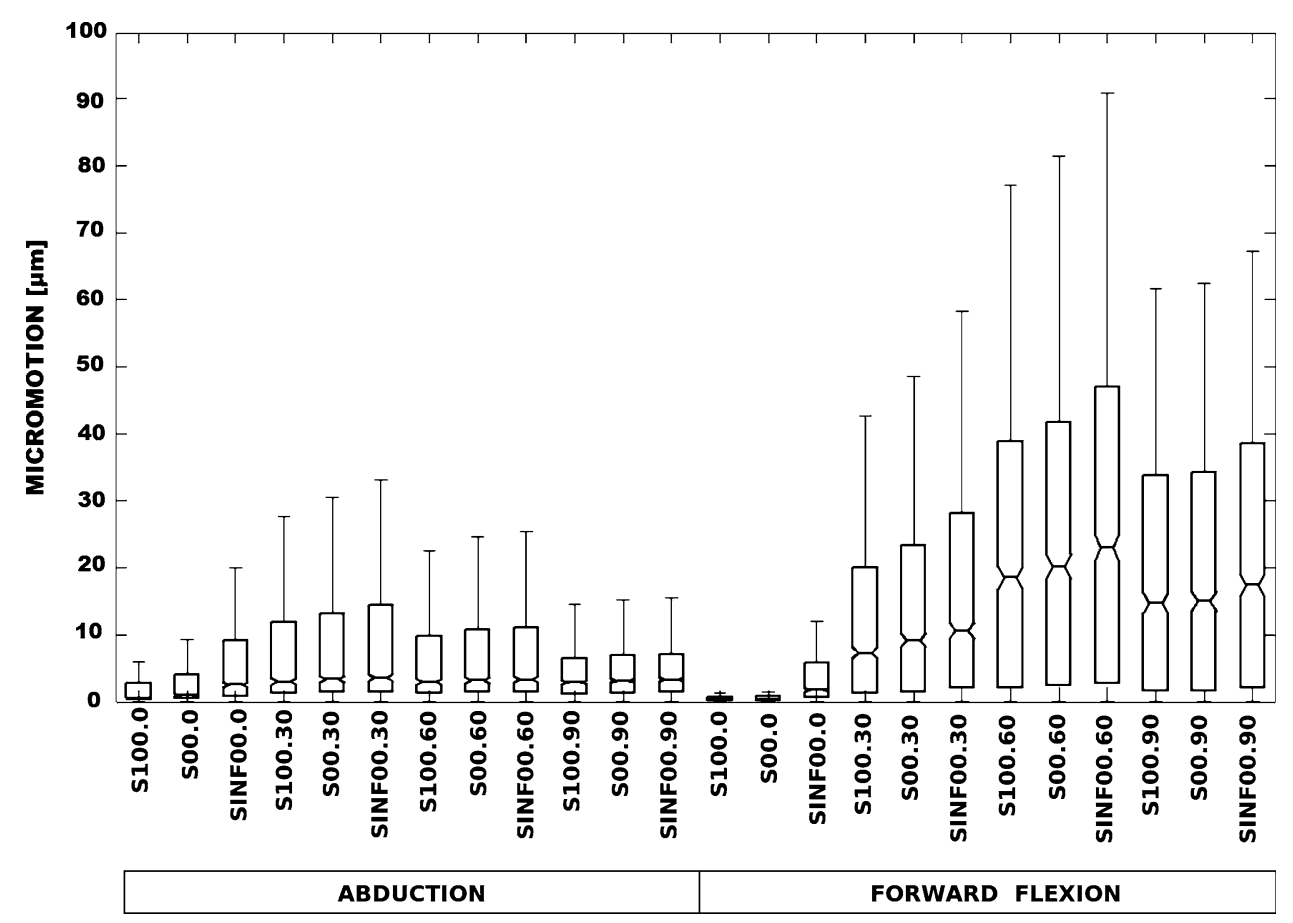

\section{Discussion}

The magnitude of the JRF increased with the rising of the arm in all the rotator cuff conditions. This increment did not change considerably between the different rotator cuff conditions. However, the JRF application points were closer to or at the glenoid component's rim when a total dysfunction of supraspinatus and infraspinatus was simulated (SINF00).

The largest values and dispersions of interface micromotions were found during arm forward flexion, $60^{\circ}$ being the worst position (i.e. creating the largest micromotions). This also coincides with the JRF being applied on the posterior rim of the glenoid, making clear that micromotions were not only linked to the magnitude but also, and more strongly, to the application point of the JRF and consequently to the arm positions.

On one hand, our results suggested that initial implant stability of the studied glenoid implant was hardly affected by degeneration of the supraspinatus. This can be explained by the high conformity of the humeral head and the glenoid component used in this study. The glenoid component kept the humeral head in its position and as a result the JRF did not change considerably when the supraspinatus force was changed. On the other hand, the results were different when failures of the supraspinatus and infraspinatus were simulated. This apparently compromised the rotator cuff's function and the fully-conforming implant was no longer 

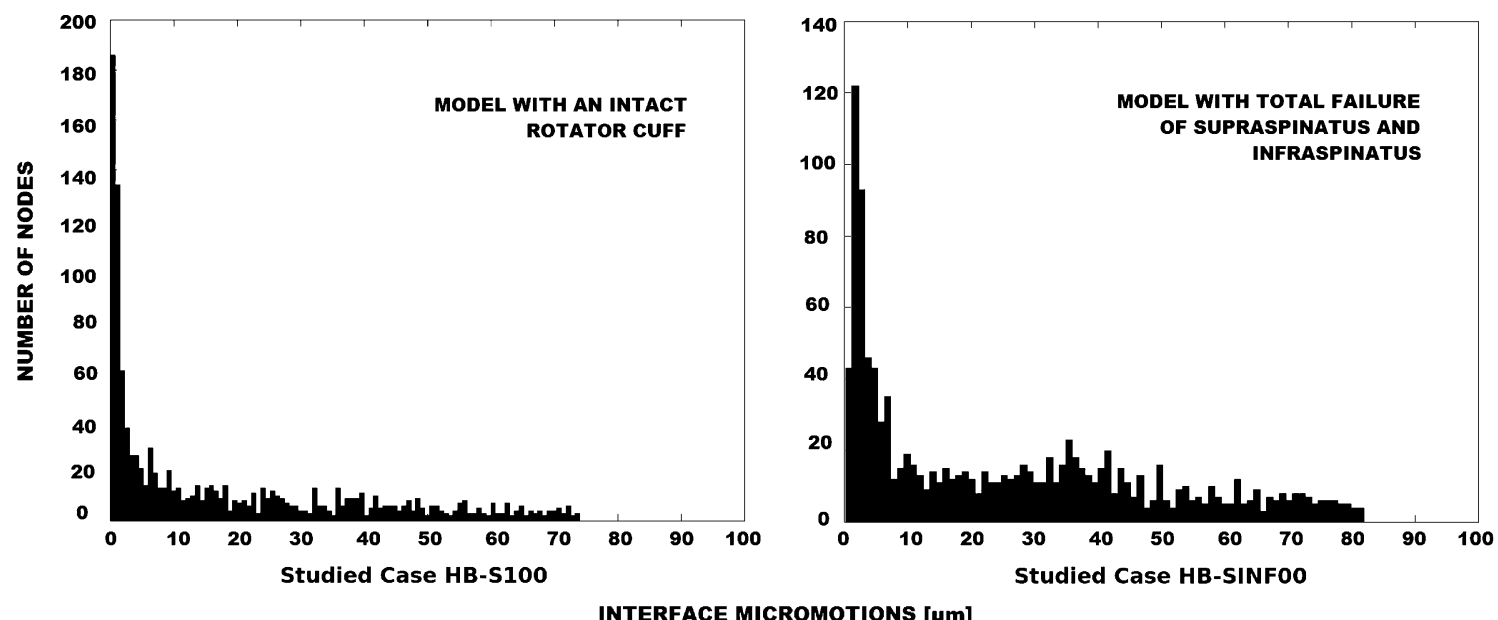

Fig. 3 Histograms of the interface micromotions for two of the studied cases with healthy bone (HB-S100 and HB-SINF00)

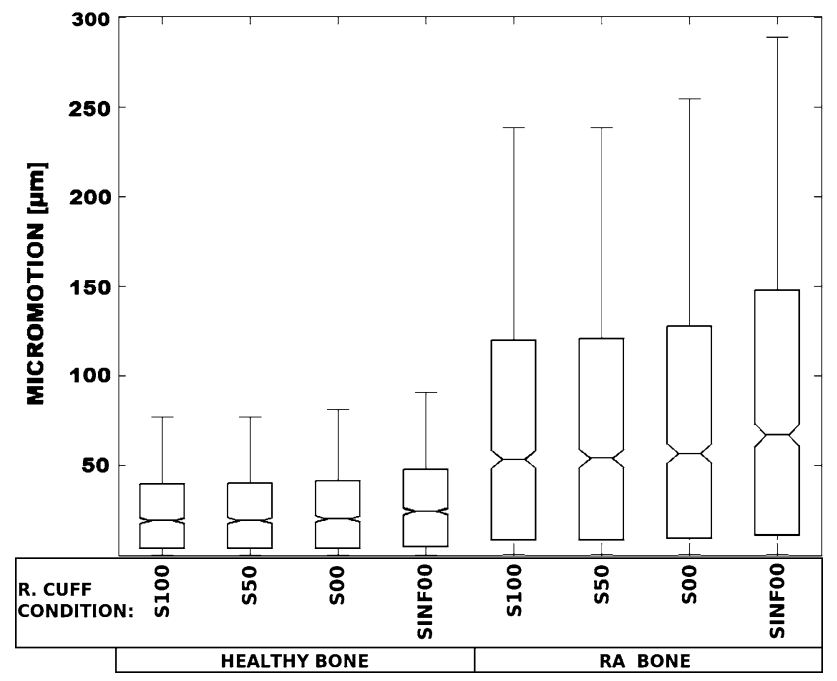

Fig. 4 Box plot of the interface micromotions for each of the studied cases. In healthy bone, micromotions in the condition SINF00 were significantly larger to the ones found in conditions S100 and S50. In RA bone, micromotions in the condition SINF00 were significantly larger to the micromotions in all the other cuff conditions. Micromotions in RA bone were significantly larger than in healthy bone

able to keep the JRF near the center of the prosthetic glenoid. Magermans et al. [14] using the DSEM to study possible tendon transfers also found limitations during arm forward flexion with deficient supraspinatus and infraspinatus muscles. This seems consistent with clinical results that did not show influence of a small defect isolated to the supraspinatus on the clinical outcome of a TSA, although larger defects comprising supraspinatus and infraspinatus did $[8,11]$. It is interesting that Figgie et al. reported that patients with a fibrotic rotator cuff or cuff deficiencies rarely gained functional arc of forward flexion; this seems to match with our findings: forward flexion produced larger implant instability.
The rotator cuff deficiency may produce an upward migration of the humeral head [15] and subsequently a shift of the JRF application point at the glenoid component during abduction, forward flexion, or extension. It is suggested that these off-center rocking movements might contribute to stress the component anchorage in the bone; this is known as the rocking horse effect. Clinical trails have pointed to this effect as a reason of the glenoid failure $[4,9]$. These studies focused on cemented and non-conforming components. In our study, we saw how eccentric loads also influence the cementless components fixation, boosting interface micromotions. However, a failure of the rotator cuff was not the only reason of significantly larger interface micromotions. Arm motions (forward flexion) and poor bone quality had even greater effect on increasing interface micromotions.

A posterior and eccentric JRF (as present during forward flexion) produced larger interface micromotions than loading at the superior rim. This is complementary to studies in which it is seen that a superior migration of the humeral head loads the superior rim, resulting in the component loosening [9, 23]. In clinics, this proximal migration is a static measurement with the arm in rest, but a posterior eccentric loading may occur with forward flexion of the arm. In this study, a progressive dysfunctional rotator cuff produced a superior displacement of the JRF. However, it was not superior eccentric loading that threatened the implant stability, but the posterior eccentric loading during arm forward flexion. In the current study, this was probably also a consequence of the lack of bone support at the lateral sides of the component's back, as it is seen in Fig. 5.

Models in which RA cases were simulated (RAB) had considerably larger interface micromotions. However, we must be aware that the simulated bone quality reduction was extreme (the Young's moduli were reduced to $50 \%$ 
Fig. 5 Detail of the FE model with (left) and without (right) glenoid component. Note that bone does not cover the whole component's back. This might be the cause of large micromotions when the component is loaded at or near the component's posterior rim

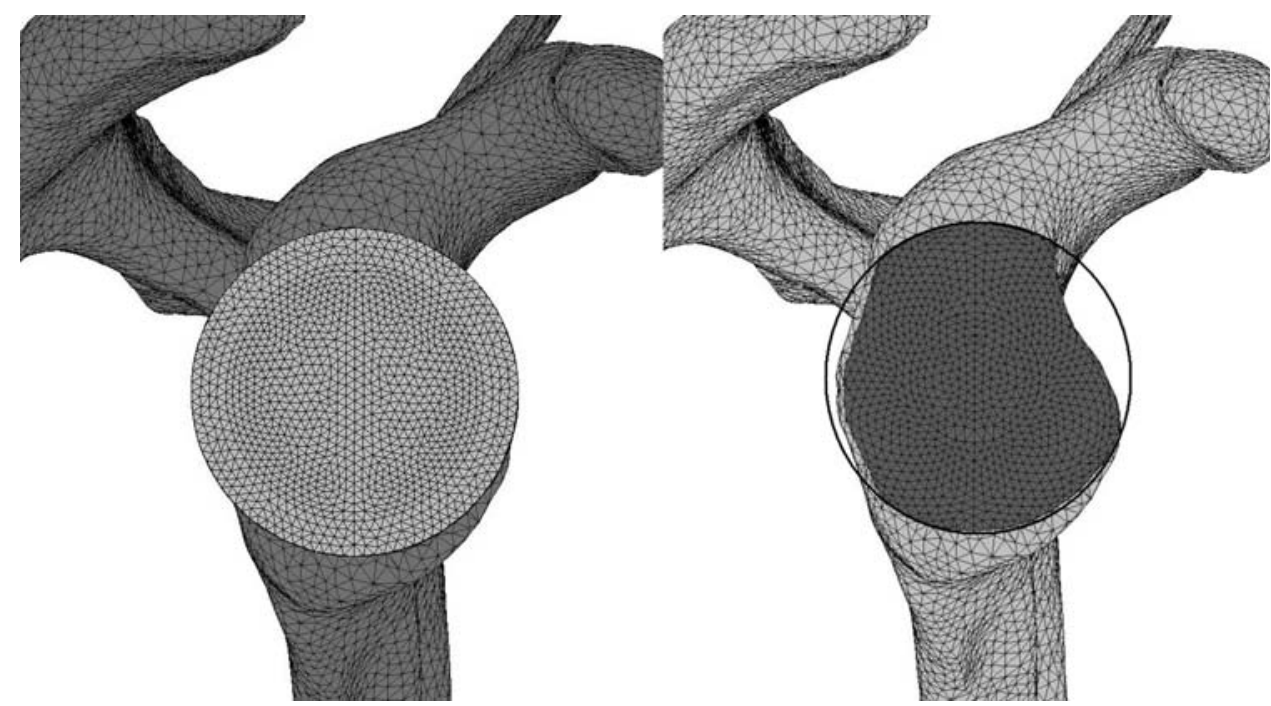

and $10 \%$ for cortical and trabecular bone, respectively). Large micromotions may not only induce instability of the component but also produce a subsequent poor clinical outcome. Furthermore, they are also related with poor bone ingrowth around the porous coating of the implant. With fully dysfunctional supraspinatus and infraspinatus micromotions became significantly larger and, consequently, a diseased rotator cuff may also jeopardize the bone ingrowth process and mid- and long-term fixation.

These possible consequences of larger interface micromotions resulting from diseased bone and a dysfunctional rotator cuff are in agreement with clinical follow-up studies that reported unsatisfactory clinical outcome in those cases $[1,7]$.

The presented FE models had several limitations and assumptions. First, the models assumed that bone is a linear elastic and isotropic material. Second, the glenohumeral force was modeled as a single force instead of a distributed load on the polyethylene surface. This affects the stresses in the polyethylene cup but we did not observe differences in terms of the interface micromotions in preliminary models in which the glenohumeral force was distributed among several nodes of the articular surface. In addition, the muscle forces given by the DSEM and used by our FE models are considered very similar to the physiological forces. Qualitative and more recently quantitative validations have shown that the DSEM is able to predict muscle activation and the JRF with a good level of correlation with respect to in-vivo measurements $[2,6,16,22]$. The use of the same scapula in both the FE model and the DSEM avoided any error due to force scaling. Third, the effect of the central screw of the glenoid component was modeled by tying the interface nodes between bone and prosthesis. This simplification influenced the estimation of stresses inside the bone and the general level of the interface micromotions. We would expect a stiffer fixation between the component and the bone if a more accurate representation of the screw were implemented. This would result in general smaller micromotions over the entire interface and the reported differences between some of the studied cases could become not significant. At last, one limitation of these models was the absence of a time-dependant bone process (i.e. bone ingrowth and remodeling) which limits the results to a time shortly after surgery.

\section{Conclusions}

According to our three dimensional simulation model, which included realistic bone properties (healthy and rheumatoid arthritic) and different muscle conditions, a small degeneration of the rotator cuff, which was represented by a reduction in the supraspinatus muscle force, does not hamper the initial fixation of a cementless glenoid fixation; but a more extended defect, which includes supraspinatus and infraspinatus muscles, results in larger micromotions. Eccentric loading in a cementless glenoid component increases the interface micromotions and may hamper the bone ingrowth process, increasing the risk of loosening.

Finally, this study is relevant to orthopedic surgeons and physical therapists. Surgeons could expect a good initial stability of a cementless glenoid component if the glenoid bone stock is adequate and a possible dysfunction of the rotator cuff is limited to the supraspinatus muscle/tendon. Physical therapists may conclude that patients with cementless glenoid components need a special post-surgery rehabilitation protocol to avoid arm motions that hamper the initial mechanical stability of the implant. Further research is necessary for including bone processes as bone 
ingrowth and (re)modeling. This could give us more insight about which variables are the most important in the longterm after a TSA.

Acknowledgments Authors would like to acknowledge prof. Frans van der Helm, prof. Rob Nelissen, Andriy Andreykiv and Frans Steenbrink for their valuable help during the different phases of this study. To Roman Nassutt (ESKA Implants, Lübeck, Germany) for supplying material properties of the Multiplex glenoid implant. D.R. Suarez is supported by Reumafonds, J.C. van der Linden is supported by the Technology Foundation STW (RPG6294).

Open Access This article is distributed under the terms of the Creative Commons Attribution Noncommercial License which permits any noncommercial use, distribution, and reproduction in any medium, provided the original author(s) and source are credited.

\section{References}

1. Barrett WP, Thornhill TS, Thomas WH, Gebhart EM, Sledge CB (1989) Nonconstrained total shoulder arthroplasty in patients with polyarticular rheumatoid arthritis. J Arthroplasty 4:91-96. doi: 10.1016/S0883-5403(89)80058-0

2. Bergmann G, Graichen F, Bender A, Kaab M, Rohlmann A, Westerhoff P (2007) In vivo glenohumeral contact forcesmeasurements in the first patient 7 months postoperatively. J Biomech 40:2139-2149. doi:10.1016/j.jbiomech.2006.10.037

3. Botha CP, Post FH (2008) Hybrid scheduling in the DeVIDE dataflow visualisation environment. Presented at simulation and visualization 2008 (SimVis 2008), Magdeburg

4. Cofield RH (1984) Total shoulder arthroplasty with the Neer prosthesis. J Bone Joint Surg Am 66:899-906

5. Dalstra M, Frich LH, Sneppen O (1996) The loss of load-bearing capability in rheumatoid glenoids (Abstract). Presented at 10th Conference of the European Society of biomechanics, Leuven, Belgium

6. de Groot JH, Rozendaal LA, Meskers CG, Arwert HJ (2004) Isometric shoulder muscle activation patterns for 3-D planar forces: a methodology for musculo-skeletal model validation. Clin Biomech (Bristol, Avon) 19:790-800. doi:10.1016/j. clinbiomech.2004.05.013

7. Edwards TB, Boulahia A, Kempf JF, Boileau P, Nemoz C, Walch $G$ (2002) The influence of rotator cuff disease on the results of shoulder arthroplasty for primary osteoarthritis: results of a multicenter study. J Bone Joint Surg Am 84-A:2240-2248

8. Figgie HE 3rd, Inglis AE, Goldberg VM, Ranawat CS, Figgie MP, Wile JM (1988) An analysis of factors affecting the longterm results of total shoulder arthroplasty in inflammatory arthritis. J Arthroplasty 3:123-130. doi:10.1016/S0883-5403(88) 80077-9
9. Franklin JL, Barrett WP, Jackins SE, Matsen FA 3rd (1988) Glenoid loosening in total shoulder arthroplasty. Association with rotator cuff deficiency. J Arthroplasty 3:39-46. doi:10.1016/ S0883-5403(88)80051-2

10. Frinch H (1994) Strength and Structure of Glenoidal Bone. Århus University, Denmark

11. Iannotti JP, Norris TR (2003) Influence of preoperative factors on outcome of shoulder arthroplasty for glenohumeral osteoarthritis. J Bone Joint Surg Am 85-A:251-258

12. Kaptein BL, van der Helm FC (2004) Estimating muscle attachment contours by transforming geometrical bone models. J Biomech 37:263-273. doi:10.1016/j.jbiomech.2003.08.005

13. Magermans DJ, Chadwick EK, Veeger HE, Rozing PM, van der Helm FC (2004) Effectiveness of tendon transfers for massive rotator cuff tears: a simulation study. Clin Biomech (Bristol, Avon) 19:116-122. doi:10.1016/j.clinbiomech.2003.09.008

14. Magermans DJ, Chadwick EK, Veeger HE, van der Helm FC, Rozing PM (2004) Biomechanical analysis of tendon transfers for massive rotator cuff tears. Clin Biomech (Bristol, Avon) 19:350357. doi:10.1016/j.clinbiomech.2003.11.013

15. Neer CS 2nd, Craig EV, Fukuda H (1983) Cuff-tear arthropathy. J Bone Joint Surg Am 65:1232-1244

16. Nikooyan AA, Veeger HEJ, van der Helm FCT, Westerhoff P, Graichen F, Bergmann G (2008) Comparing model-predicted gh-joint contact forces by in-vivo measured forces. J Biomech 41:S145. doi:10.1016/S0021-9290(08)70145-3

17. Pilliar RM, Lee JM, Maniatopoulos C (1986) Observations on the effect of movement on bone ingrowth into porous-surfaced implants. Clin Orthop Relat Res 208:108-113

18. Ramamurti BS, Orr TE, Bragdon CR, Lowenstein JD, Jasty M, Harris WH (1997) Factors influencing stability at the interface between a porous surface and cancellous bone: a finite element analysis of a canine in vivo micromotion experiment. J Biomed Mater Res 36:274-280. doi:10.1002/(SICI)1097-4636(199708) 36:2<274::AID-JBM17>3.0.CO;2-G

19. Rice JC, Cowin SC, Bowman JA (1988) On the dependence of the elasticity and strength of cancellous bone on apparent density. J Biomech 21:155-168. doi:10.1016/0021-9290(88)90008-5

20. Schaffler MB, Burr DB (1988) Stiffness of compact bone: effects of porosity and density. J Biomech 21:13-16. doi:10.1016/00219290(88)90186-8

21. Uhthoff HK, Matsumoto F, Trudel G, Himori K (2003) Early reattachment does not reverse atrophy and fat accumulation of the supraspinatus-an experimental study in rabbits. J Orthop Res 21:386-392. doi:10.1016/S0736-0266(02)00208-5

22. van der Helm FC (1994) A finite element musculoskeletal model of the shoulder mechanism. J Biomech 27:551-569. doi:10.1016/ 0021-9290(94)90065-5

23. Williams GR Jr, Rockwood CA Jr (1996) Hemiarthroplasty in rotator cuff-deficient shoulders. J Shoulder Elbow Surg 5:362367. doi:10.1016/S1058-2746(96)80067-X 